\title{
Prevention of cataract in diabetic mice by topical pyruvate
}

This article was published in the following Dove Press journal:

Clinical Ophthalmology

17 August 2011

Number of times this article has been viewed

KR Hegde ${ }^{1,3}$

S Kovtun'

SD Varma ${ }^{1,2}$

'Ophthalmology and Visual Sciences, ${ }^{2}$ Biochemistry and Molecular Biology, University of Maryland School of Medicine, ${ }^{3}$ Coppin State University, Department of Natural Sciences, Baltimore, MD, USA
Correspondence: Shambhu D Varma Ophthalmology and Visual Sciences, Biochemistry and Molecular Biology, MSTF 5-00A, I0 South Pine Street, University of Maryland School of Medicine, Baltimore, MD 2I20I, USA

$\mathrm{Tel}+$ I 4107063395

Fax + 4107067057

Emailsvarm00।@umaryland.edu
Background: It has been previously reported that oral administration of sodium pyruvate inhibits oxidative stress and cataract formation in diabetic animals. With a view to exploring the clinical usefulness of these findings, this study examined its preventive effect when administered topically as an eye drop.

Methods: Diabetes was induced by intraperitoneal injections of streptozotocin. At the onset of diabetes, an eye drop preparation containing $2.5 \%$ sodium pyruvate was administered six times a day at 90-minute intervals. Treatment was continued for 6 weeks. Cataract formation was monitored ophthalmoscopically after mydriasis with $1 \%$ tropicamide eye drops. Subsequently, the treated and untreated diabetic animals and the age-matched normal controls were euthanized, their eyes enucleated, and the lenses isolated for biochemical assessment of protein glycation and glutathione levels.

Results: Treatment with pyruvate eye drops was found to be significantly effective in inhibiting protein glycation. Glutathione levels were also better maintained. In addition, ophthalmoscopic examination revealed that the incidence of cataract in the pyruvate-treated group was only $12 \%$ as compared with the untreated diabetics in whom the incidence was $73 \%$. Cataracts at this stage were largely equatorial.

Conclusion: The results demonstrate that topical application of pyruvate can potentially be useful in attenuating or preventing cataract formation induced by diabetes and other conditions of oxidative stress.

Keywords: pyruvate eye drops, diabetic cataract, protein glycation, oxidative stress

\section{Introduction}

Cataract is one of the most common causes of vision impairment and blindness with significant adverse effects on the quality of life of the individual. ${ }^{1}$ Even though surgical treatment is successful in restoring vision, the incidence of this disease is so high that surgery alone has not been able to eradicate cataract blindness, mainly because of replacement of old cases with new ones as well as the increase in longevity. Therefore, it is highly desirable to explore the possibility of preventing or treating cataracts pharmacologically.

Several previous studies strongly suggest that oxidative stress consequent to excessive generation of oxygen free radicals is one of the significant factors involved in the pathogenesis of this aging disease. ${ }^{2-5}$ This is attributable to decreased activity of endogenous antioxidant defense enzymes, viz., catalase, superoxide dismutase, and glutathione peroxidase, as well as accumulation of tryptophan degradation products, such as kynurenine. The latter can induce oxidative stress by acting as a photosensitizer 
and consequently generate excessive reactive oxygen species during photopic vision. ${ }^{6,7}$ The consequent increase in reactive oxygen species leads to oxidative modifications of cellular constituents, such as the enzymatic and nonenzymatic proteins, lipids, nucleic acids, and the pyridine nucleotides, with deleterious effects. Reactive oxygen species generation is further enhanced in certain diseases such as diabetes, wherein the cataract is known to start at an earlier age and progresses relatively faster than in nondiabetics. ${ }^{8-10}$ Enhancement of the process in diabetes has been attributed to increased generation of reactive oxygen species by metal-catalyzed auto-oxidation of glucose present at higher concentrations. In addition to increased reactive oxygen species generation, the process is accompanied by generation of highly reactive dicarbonyls ${ }^{11-13}$ which modify protein structures and functions by glycation, leading eventually to formation of advanced glycation endproducts and the high molecular weight protein aggregates characteristic of cataracts. Glycated proteins can by themselves generate oxyradical species, thereby perpetuating the oxidative stress even further. The reactive oxygen species generated are known to be highly effective in causing - SH oxidation of several enzymes involved in the maintenance of tissue physiology, such as glyceraldehyde-3-phosphate dehydrogenase, a key glycolytic enzyme. Metabolic inhibition in diabetes is reflected also by a decreased respiratory quotient, ie, $\mathrm{CO}_{2} / \mathrm{O}_{2}$, first demonstrated by Richardson and Levine, ${ }^{14}$ showing decreased utilization of molecular oxygen through normal metabolic pathways and its consequent diversion to auto-oxidation reactions known to proceed monovalently. In addition to its adverse effects on metabolic enzymes, reactive oxygen species inhibit membrane transport activity by inactivating $\mathrm{Na}^{+}-\mathrm{K}^{+}$ATPase, ${ }^{15,16}$ the enzyme responsible for maintaining the lens in a deturgesced state by regulating active transport of cations. Hence the deleterious effects of reactive oxygen species are varied in nature. Therefore, it is expected that the use of reactive oxygen species scavengers would be beneficial in preventing oxidative stress-induced damage to the lens and cataract formation.

Previous studies, including those from our laboratory, have shown that nutritional antioxidants, such as ascorbate and vitamin $\mathrm{E}$, are effective in preventing cataract formation in animal models of oxidative stress as well as diabetes. ${ }^{17,18}$ However, the clinical usefulness of these compounds becomes limited due to their tendency to become pro-oxidant following their reaction with reactive oxygen species. ${ }^{19}$ Therefore, we believe that the effectiveness of such scavengers could be enhanced if they are able to provide bioenergetic support to the tissue simultaneously by facilitating ATP generation. In this regard, we have previously shown that pyruvate is highly effective in preventing oxidative stress to the lens in vitro, ${ }^{16}$ as well as in preventing actual cataract formation in vivo when administered orally to rats or mice with experimentally-induced diabetes. ${ }^{20,21}$ The latter is the preferred model for such studies due to its low aldose reductase activity in the lens, similar to that in the human lens. ${ }^{22}$ The ability of pyruvate in preventing cataract formation is also reflected biochemically in terms of the maintenance of levels of glutathione and inhibition of protein glycation in the lenses of diabetic mice. Its beneficial effect was further apparent in terms of prevention of diabetes-induced apoptotic changes in the lens. ${ }^{23}$ Encouraged by these findings, we undertook the present investigation to examine the possibility of inhibiting diabetic cataract formation by topical treatment with pyruvate eye drops, a pharmacologically more acceptable and often more effective way of treating eye diseases.

\section{Materials and methods}

CD-1 mice (body weight $25 \mathrm{~g}$ ) obtained from Charles River Laboratories (Wilmington, MA) were used in this research. The procedures used for animal handling and euthanasia followed the Association for Research in Vision and Ophthalmology Statement for the Use of Animals in Research and were approved by the Institutional Animal Care and Use Committee. Diabetes was induced by intraperitoneal injection of streptozotocin $40 \mathrm{mg} / \mathrm{kg}$ body weight for five consecutive days. Onset of hyperglycemia was ascertained one week following streptozotocin by determining the levels of blood glucose with a glucometer using tail blood. Animals with blood glucose of $350-400 \mathrm{mg} / \mathrm{dL}$ were included in the study. The diabetic animals were divided into two groups whereby one group was left untreated (control diabetics) and the second group was treated with $2.5 \%$ sodium pyruvate eye drops six times a day, the treatment being started immediately following the establishment of diabetes and continued until 6 weeks. The eye drops were freshly prepared daily by dissolving $2.5 \mathrm{~g}$ sodium pyruvate in a solution containing $0.3 \%$ hydroxypropyl methyl cellulose (3500-5600 cps, pH about 7) as a wetting agent. Development of cataract was monitored by ophthalmoscopy following mydriasis with $1 \%$ tropicamide eye drops. After six weeks of treatment, the untreated and treated diabetic animals as well as the age-matched normal controls were euthanized, their eyes enucleated, and the lenses isolated for biochemical analyses.

\section{Penetration of sodium pyruvate in aqueous humor}

That the pyruvate administered topically does penetrate through the cornea was ascertained by determining its 
level in the aqueous humor following instillation in normal mice before the start of the actual studies. This was done as follows. After induction of anesthesia by intraperitoneal ketamine:xylazine $(80 \mathrm{mg} / \mathrm{kg}$ body weight ketamine, $10 \mathrm{mg} / \mathrm{kg}$ body weight xylazine), about $25 \mu \mathrm{L}$ of the above eye drop preparation was administered in the cul-de-sac of the mouse eye. At 30 minutes after administering the eye drops, the eye was rinsed with normal saline and aqueous humor aspirated using a $25 \mathrm{G} \mathrm{5/8}$ disposable needle attached to an insulin syringe and analyzed for its pyruvate content. The basal level of pyruvate in the aqueous humor was determined in the anesthetized animals that did not receive the eye drops. Pyruvate concentration was determined enzymatically by mixing the sample with nicotinamide adenine dinucleotide and lactate dehydrogenase reagent and monitoring spectrophotometrically the decrease in absorption (at $340 \mathrm{~nm}$ ) due to reduction of pyruvate to lactate, as reported previously. ${ }^{24}$

Biochemical analyses of the lenses obtained from normal, untreated, and pyruvate-treated diabetic groups were done as follows: An aqueous extract of the tissue was prepared by homogenization in $0.5 \mathrm{~mL}$ distilled $\mathrm{H}_{2} \mathrm{O}$ and centrifugation at 14,000 rpm. The supernatant was used for determining glutathione and glycated protein content.

\section{Determination of lens glutathione content}

Glutathione was determined in an aliquot of the above supernatant using Ellman's reaction. ${ }^{25}$ After precipitating the proteins present therein by addition of trichloroacetic acid to a final concentration of $5 \%$, the sample was centrifuged. About $100 \mu \mathrm{L}$ of the supernatant so obtained was reacted with $100 \mu \mathrm{L}$ of Ellman's reagent after neutralization with $300 \mu \mathrm{L}$ of $0.6 \mathrm{M} \mathrm{Na}_{2} \mathrm{HPO}_{4}$. The yellow color developed due to the formation of thionitrobenzoate was then read at $412 \mathrm{~nm}$.

\section{Measurement of protein glycation}

Protein glycation was measured as follows. ${ }^{20,21}$ The content of water-soluble proteins in the aqueous supernatant prepared as described earlier was determined by Bradford's method using BioRad protein reagent. An aliquot of the supernatant containing $100 \mu \mathrm{g}$ of the proteins was then loaded on a boronate affinity chromatographic column (Sigma kit 442-B; Sigma-Aldrich, St Louis, MO) which binds glycated proteins containing cis-diols of the sugar bound to the protein. After 10 minutes of reaction on the column, the unglycated proteins were eluted with phosphate buffer $\mathrm{pH} 9$ (Sigma 442-3). The glycated protein was then eluted with $50 \mathrm{mM}$ sorbitol in $0.4 \mathrm{~N}$ $\mathrm{NaOH}$. Total proteins were determined in the two fractions by Bradford's method, and the percentage of glycated protein was calculated.

\section{Results}

Previous studies from our laboratory have demonstrated the effectiveness of pyruvate in preventing cataract formation in diabetic animals. The preventive effect was evaluated biochemically as well as morphologically. Pyruvate was administered systemically by mixing it in the diet. However, given that topical treatment is the preferred mode of treatment for ocular diseases, it was considered desirable to determine the efficacy of topical instillation of pyruvate. Therefore, initial experiments were done to examine if adequate levels of the compound in the aqueous humor are indeed attained following such treatment. The level of pyruvate in the aqueous increases from a basal level of about $0.2 \mathrm{mM}$ to about $1.2 \mathrm{mM}$ at 30 minutes following instillation of $2.5 \%$ sodium pyruvate eye drops in the normal mouse eye. It decreases to the basal level by 60 minutes. Instillation of such an eye drop preparation in the mouse eye six times daily was found to be highly effective in inhibiting the onset of cataract formation. The earliest sign of cataract in diabetic mice consisted of the appearance of a peripheral ring of cloudiness in the lens, visible ophthalmoscopically at about 5-6 weeks of diabetes. However, such opacity was difficult to discern by standard slit lamp examination due to the convexity of the lens and peripheral location of the opacity. As shown in Table 1, $73 \%$ of the eyes in the untreated diabetic group had such cataracts. Interestingly, in the diabetic animals receiving pyruvate eye drops, only $12.5 \%$ of the eyes had developed early cataractous changes.

Inhibition of cataract formation by topical pyruvate treatment was due to inhibition of oxidative stress, as indicated by measurements of glutathione levels. As shown in Figure 1, the level of glutathione, the primary antioxidant reserve of the tissue, decreased significantly (by about $50 \%$ ) in the diabetic mouse lens to about $1.2 \mu \mathrm{mol} / \mathrm{g}$ from a normal value of approximately $2.4 \mu \mathrm{mol} / \mathrm{g}$. Pyruvate administration prevented this decrease, the levels in this case being approximately $2.3 \mu \mathrm{mol} / \mathrm{g}$, which is close to the normal. In addition to the direct effect of reactive oxygen species on glutathione, an additional mechanism by which glucose induces lens opacity is structural modification of the lens proteins by nonenzymatic glycation and subsequent formation of advanced glycation end-products and high molecular weight aggregates with decreased solubility. As shown in Figure 2, the level of glycated proteins in the normal lens was about $1.5 \%$, which increased to nearly $9 \%$ in the 
Table I Effect of pyruvate eye drops on the development of cataract in diabetic mice was assessed by ophthalmoscopy after mydriasis. As shown, the number of cataracts in the untreated diabetic group was significantly higher as compared with the pyruvate-treated group. Duration of diabetes was 6 weeks

\begin{tabular}{llll}
\hline & Normal & Diabetes (6 weeks) & $\begin{array}{l}\text { Diabetes (6 weeks) }+ \\
\text { 2.5\% pyruvate eye drops }\end{array}$ \\
\hline Eyes with cataract $(n)$ & $0 / 12$ eyes & $16 / 22$ eyes $(73 \%)$ & $3 / 24$ eyes $(12.5 \%)$ \\
\hline
\end{tabular}

diabetic lens. Treatment with pyruvate eye drops substantially prevented such an increase, the level in this case being only about $3 \%$.

\section{Discussion}

Diabetes is one of the significant risk factors for development of cataract. This is apparent in several studies showing that the incidence of cataracts in the diabetic population is higher than in nondiabetics. In addition, the onset of cataract occurs at an earlier age and progresses at a faster pace in diabetics. One of the important factors involved in the pathogenesis of cataracts is induction of oxidative stress consequent to excessive intraocular generation of reactive oxygen species triggered by the high levels of glucose in the extracellular as well as intracellular fluids. The latter is especially true in relatively insulin-insensitive tissues such as the lens. We have previously shown that such stress induced in the lens by diabetes is preventable by oral administration of pyruvate, a potent oxyradical scavenger and a metabolic agonist. The anticataractogenic effect of systemic treatment with pyruvate encouraged us to undertake further studies examining its potential effectiveness against cataract development by topical administration, which is the preferred route for such treatment clinically. It is expected that a higher level of the

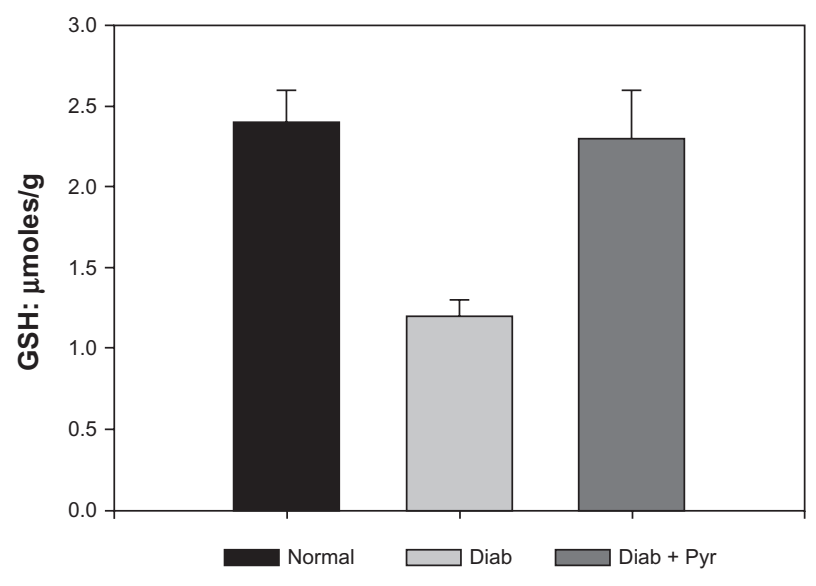

Figure I Levels of glutathione in the diabetic mouse lens. Glutathione was determined in the acid extract of the tissue as described in the text to estimate the effect of topical pyruvate.

Note: Data represent mean \pm standard deviation $(n=6 ; P<0.001)$ between normal and untreated diabetic groups as well as between the untreated and pyruvatetreated diabetic groups. compound can be achieved in the aqueous humor when it is administered by this route. Indeed adequate levels of pyruvate were attained in the aqueous humor (about $1.2 \mathrm{mM}$ ) following its topical instillation. We have recently shown that it also penetrates the human cornea to a significant extent when administered topically. ${ }^{26}$ The levels so attained in the aqueous humor were found to be significantly effective in inhibiting oxidative stress at the lens, apparent by the maintenance of glutathione levels. This effect is attributable primarily to the ability of pyruvate to scavenge reactive oxygen species. The reaction of pyruvate with all such species has been shown to be thermodynamically highly favorable, including its reaction with the hydroxyl radical. ${ }^{27,28}$

Pyruvate treatment was also effective in inhibiting protein glycation. It has previously been shown to exert this effect through competitive inhibition of glycation by glucose. The carbonyl group of pyruvate competes with the glucose carbonyl for reaction with the $-\mathrm{NH}_{2}$ protein group for the formation of the initial Schiff's adduct. Because the carbonyl group of glucose and its derivatives remains masked in the hemiacetal configuration, it is less freely available for reacting with the $-\mathrm{NH}_{2}$ protein versus the pyruvate carbonyl which is exposed to react. In addition, due to the absence of vicinyl hydroxyl groups in the Schiff's base formed between $-\mathrm{NH}_{2}$

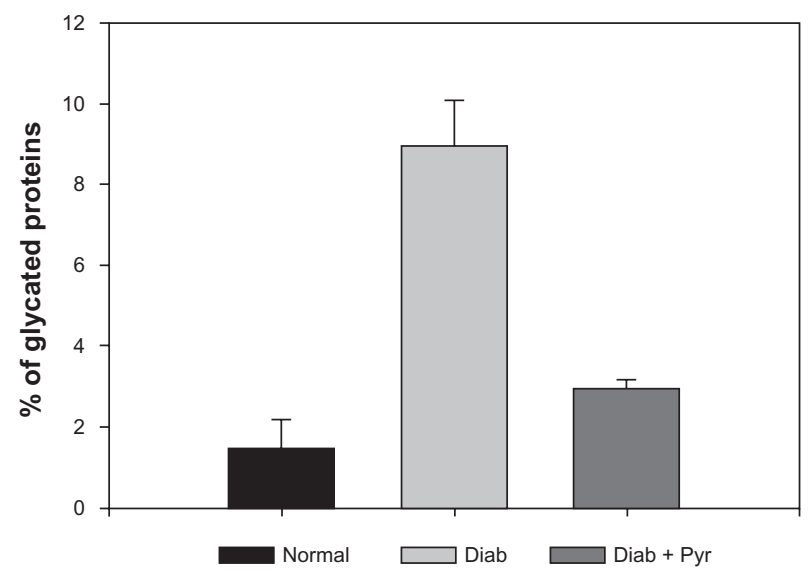

Figure 2 Effect of pyruvate eye drops on the extent of protein glycation in the diabetic mouse lens: Glycated proteins were determined in the aqueous lens extract by affinity chromatography using a boronate column.

Note: Results represent mean \pm standard deviation $(n=6, P<0.001)$ between normal and untreated diabetic groups as well as between untreated and pyruvatetreated diabetic groups. 
protein and pyruvate, the complex does not undergo Amadori rearrangement and hence formation of advanced glycation end-products is prevented.

The protective effect of pyruvate eye drops against biochemical damage to the lens in diabetic animals was hence similar to that observed with its oral administration. We believe that the effectiveness of topical pyruvate can be further enhanced by using its ester, ethyl pyruvate, shown recently by us to attain significantly higher concentrations than its sodium salt in the aqueous humor and lens. ${ }^{24}$ Further studies examining its effectiveness in preventing cataract formation in diabetic animals even until later stages of the disease are in progress.

\section{Acknowledgments}

The authors are grateful for the financial support of the National Eye Institute, National Institutes of Health.

\section{Disclosure}

The authors report no conflicts of interest in this work.

\section{References}

1. Resnikoff S, Pascolini D, Etya'ale D, et al. Global data on visual impairment in the year 2002. Bull World Health Organ. 2004;82:844-851.

2. Varma SD, Srivastava VK, Richards RD. Photoperoxidation in lens and cataract formation: preventive role of superoxide dismutase, catalase and vitamin C. Ophthalmic Res. 1982;14:167-175.

3. Spector A, Garner WH. Hydrogen peroxide and human cataract. Exp Eye Res. 1981;33:673-681.

4. Varma SD, Ets TK, Richards RD. Protection against superoxide radicals in rat lens. Ophthalmic Res. 1977;9:421-431.

5. Bhuyan DK, Bhuyan KC. Regulation of hydrogen peroxide in eye humors. Effect of 3-amino-1H-1,2,4-triazole on catalase and glutathione peroxidase of rabbit eye. Biochim Biophys Acta. 1977;497:641-651.

6. Van Heyningen R. Photo-oxidation of lens proteins by sun light in the presence of derivatives of kynurenine isolated from human lens. Exp Eye Res. 1973;17:137-147.

7. Dillon J, Wang RH, Athertso S. Photo-chemical and photo-physical studies on human lens constituents. Photochem Photobiol. 1990;2: 849-854.

8. Harding JJ, Egerton M, van Heyningen R, Harding RS. Diabetes, glaucoma, sex, and cataract: analysis of combined data from two case control studies. Br J Ophthalmol. 1993;77:2-6.

9. Kahn HA, Leibowitz HM, Ganley JP, et al. The Framingham eye study. Association of ophthalmic pathology with single variables previously measured in the Framingham heart study. Am J Epidemiol. 1977; 106:33-41.

Clinical Ophthalmology

\section{Publish your work in this journal}

Clinical Ophthalmology is an international, peer-reviewed journal covering all subspecialties within ophthalmology. Key topics include: Optometry; Visual science; Pharmacology and drug therapy in eye diseases; Basic Sciences; Primary and Secondary eye care; Patient Safety and Quality of Care Improvements. This journal is indexed on
10. Rowe N, Mitchell P, Cumming RG, Wans JJ. Diabetes, fasting blood glucose and age-related cataract: the Blue Mountains Eye Study. Ophthalmic Epidemiol. 2000;7:103-114.

11. Wolff SP, Crabbe MJC, Thornalley PJ. The autooxidation of glyceraldehydes and other simple monosaccharides. Experientia. 1984;40: 244-246.

12. Stevens MJ, Rouzer CA, Monnier VM, Cerami A. Diabetic cataract formation and potential role of glycosylation of lens proteins. Proc Natl Acad Sci U S A. 1978;75:2918-2922.

13. Baynes JW. Role of oxidative stress in development of complications in diabetes. Diabetes. 1991;40:405-412.

14. Richardson HB, Levine SZ. Clinical calorimetry. Exercise and the respiratory quotient in diabetes. J Biol Chem. 39;66:161-183.

15. Kobatashi S, Roy D, Spector A. Sodium/potassium ATPase in normal and cataractous human lenses. Curr Eye Res. 1982;2:327-334.

16. Varma SD, Morris SM. Peroxide damage to the eye lens in vitro: prevention by pyruvate. Free Radic Res Comm. 1988;4:283-290.

17. Devamanoharan PLS, Henein M, Morris SM, Ramachandran S, Richards RD, Varma SD. Prevention of selenite cataract by vitamin C. Exp Eye Res. 1991;52:563-568.

18. Ross WM, Creighton MOP, Stewart-DeHaan PJ, Sanwal M, Hirst M, Trevithick JR. Modelling cortical cataractogenesis. 3. In vivo effects of vitamin E on cataractogenesis in diabetic rats. Can J Ophthalmol. 1982;17:61-66.

19. Nagaraj RH, Monnier VM. Protein modification by the degradation products of ascorbate: formation of a novel pyrrole from the Maillard reaction of L-threose with proteins. Biochim Biophys Acta. 1995;1253: 75-84.

20. Zhao W, Devamanoharan PS, Henein M, Ali AH, Varma SD. Diabetes induced biochemical changes in rat lens: attenuation of cataractogenesis by pyruvate. Diabetes Obes Metab. 2000;2:165-174.

21. Hegde KR, Varma SD. Prevention of cataract by pyruvate in experimentally diabetic mice. Mol Cell Biochem. 2005;269:115-120.

22. Varma SD, Kinoshita JH. The absence of cataracts in mice with congenital hyperglycemia. Exp Eye Res. 1974;19:577-582.

23. Hegde KR, Varma SD. Morphogenetic and apoptotic changes in diabetic cataract: prevention by pyruvate. Mol Cell Biochem. 2004;262: 233-237.

24. Hegde KR, Kovtun S, Varma SD. Intraocular penetration of pyruvate following its topical administration in mice. Mol Cell Biochem. 2010;338:87-90.

25. Ellman G L. Tissue sulphydryl groups. Arch Biochem Biophys. 1959;82: 70-77.

26. Chandra P, Hegde KR, Varma SD. Possibility of topical antioxidant treatment of cataracts: corneal penetration of pyruvate in humans. Ophthalmologica. 2009;223:136-138.

27. Ervens B, Gligorovski S, Herrmann H. Temperature dependent rate constants for hydroxyl radical reactions with organic compounds in aqueous solutions. Phys Chem Chem Phys. 2003;5:1811-1824.

28. Millouki A, Mu YJ. On atmospheric degradation of pyruvic acid in the gas phase. J Photchem Photobiol A Chem. 2003;157:295-300.

PubMed Central and CAS, and is the official journal of The Society of Clinical Ophthalmology (SCO). The manuscript management system is completely online and includes a very quick and fair peer-review system, which is all easy to use. Visit http://www.dovepress.com/ testimonials.php to read real quotes from published authors. 